\title{
Silicon intake and plasma level and their relationships with systemic redox and inflammatory markers in rheumatoid arthritis patients
}

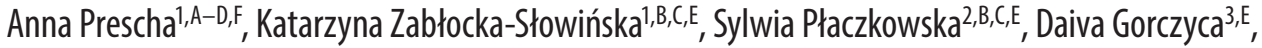 \\ Anna Łuczak ${ }^{4, B}$, Halina Grajeta ${ }^{1, E}$ \\ ${ }^{1}$ Department of Food Science and Dietetics, Wroclaw Medical University, Poland \\ 2 Diagnostics Laboratory for Teaching and Research, Wroclaw Medical University, Poland \\ $33^{\text {rd }}$ Department and Clinic of Pediatrics, Immunology and Rheumatology of Developmental Age, Wroclaw Medical University, Poland \\ ${ }^{4}$ Department and Clinic of Rheumatology and Internal Medicine, Wroclaw Medical University, Poland \\ A - research concept and design; B - collection and/or assembly of data; C - data analysis and interpretation; \\ $D$ - writing the article; $E$ - critical revision of the article; $F$ - final approval of the article
}

Address for correspondence

Anna Prescha

E-mail:anna.prescha@umed.wroc.pl

Funding sources

None declared

Conflict of interest

None declared

\section{Acknowledgements}

The authors would like to thank Prof. Piotr Wiland MD, PhD from the Department and Clinic of Rheumatology and Internal Medicine, Wroclaw Medical University, for his clinical expertise and guidance in the study design and performance. The authors also thank Joanna Kaliszczyk, MSc for her technical support.

Received on August 21, 2018

Reviewed on November 25, 2018

Accepted on March 5, 2019

Published online on April 15, 2019

Cite as

Prescha A, Zabłocka-Słowińska K, Płaczkowska S, Gorczyca D, Łuczak A, Grajeta H. Silicon intake and plasma level and their relationships with systemic redox and inflammatory markers in rheumatoid arthritis patients. Adv Clin Exp Med. 2019:28(11):1485-1494. doi:10.17219/acem/105380

DOI

10.17219/acem/105380

Copyright

Copyright by Author(s)

This is an article distributed under the terms of the

Creative Commons Attribution Non-Commercial License

(http://creativecommons.org/licenses/by-nc-nd/4.0/)

\section{Abstract}

Background. The nutritional significance of silicon for the human body is highlighted by a continually growing body of evidence. In conditions of excessive reactive oxygen species and upregulated immune response, silicon has been observed to provide benefits, but its role in redox and inflammatory status has not yet been examined in rheumatoid arthritis (RA).

Objectives. The aim of this study was to assess the relationship of silicon intake and plasma level to systemic indices of redox status and inflammation in patients with RA.

Material and methods. Silicon intake and plasma levels were measured in 115 RA subjects and 129 control subjects. Serum antioxidant and oxidant levels, antioxidant enzyme activity, and albumin, uric acid, TBARS, hs-CRP, and IL-6 levels were measured and compared to the intake and plasma levels of silicon.

Results. Silicon intake and plasma silicon levels were higher in RA subjects than in the controls. In the RA group, a generally favorable correlation to redox and inflammatory markers was found for silicon in diet and in plasma; however, albumin level, smoking status, and gender interfered with these results. In the control subjects, a significant relationship was observed only between plasma silicon and non-enzymatic markers of redox status.

Conclusions. There are suggestions of silicon's involvement in managing redox and inflammatory status in RA, though further studies are warranted.

Key words: silicon, redox status, inflammatory biomarkers, rheumatoid arthritis 


\section{Introduction}

A growing body of evidence suggests that silicon is essential to the human body, supported recently by the identification of the cellular silicon influx and efflux system..$^{1,2}$ Orthosilicic acid diluted in water and soluble dietary silicates are both easily absorbed by the intestines. Solid plant foods, such as cereals and certain vegetables, provide silicon in the form of the less absorbable phytolytic silica, but the high silicon content nevertheless makes them important dietary sources of this element. ${ }^{3,4}$ The food industry utilizes silicon compounds as food additives, in the form of food-grade amorphous silica, silicates, and polymethylosiloxane, which can substantially increase the amounts of silicon in one's diet. ${ }^{5}$ The body retains silicon mainly in connective tissues, including bone, cartilage, skin, and the aortal wall. ${ }^{1}$ Orthosilicic acid circulating in the blood is thought to be predominantly unbound to protein. ${ }^{6}$

Silicon has been shown to stimulate collagen synthesis, to promote osteoblast differentiation, and to improve bone density and turnover. Silicon deficiencies may lead to abnormalities in the connective tissue and articular cartilage in rats. Serum silicon concentration positively correlates with collagen concentration in the skin and cartilage. $^{6}$ The protective role of silicon in the development of cognitive impairment and atherosclerosis has also been shown, although the latter remains controversial. ${ }^{1}$ Studies on silicon-deprived animals suggest a contribution of silicon to immune and inflammatory responses. ${ }^{7,8}$ In murine macrophages, silicon in the form of sodium metasilicate demonstrated the ability to reduce nitric oxide generation and to inhibit the gene expression of tumor necrosis factor- $\alpha$ and cyclooxygenase- $2 .{ }^{9}$ Redox status has also been shown to improve in silicon-supplemented rats with non-alcoholic steatohepatitis, including enhanced gene expression of liver antioxidant enzymes and lowered glutathione persulfide levels. ${ }^{10}$ It should also be mentioned that the role of silicon in enhancing antioxidant capacity, including antioxidant enzyme activity, has been confirmed for plants exposed to abiotic stress. ${ }^{11}$ On the other hand, hypersilicemia induced in rats by an excessive intake of metasilicate nonahydrate led to the accumulation of oxygen free radicals in the liver and kidneys and to the inhibition of superoxide dismutase and glutathione peroxidase. $^{12}$

So far, no association of dietary silicon with inflammatory autoimmune diseases has been reported. Only exposure to non-dietary silicon, e.g., crystalline silica inhalation or silicone implants in the body has been found to increase autoimmune disease risk in humans, including RA. ${ }^{13,14}$ Dietary silicon at a physiological level has in turn shown an immunomodulatory effect in rats with arthritis induced by collagen type 2 injection. ${ }^{8}$ Rheumatoid arthritis is a chronic syndrome characterized by non-specific inflammation of mainly the peripheral joints, leading to the progressive destruction of cartilage and bone.
The generation of reactive oxygen and nitrogen species at the site of inflammation has been shown to contribute to disease development. ${ }^{15}$ It has previously been reported that an impaired ability to remove oxidative stress in RA results in a disrupted systemic redox status. ${ }^{16}$ The beneficial activity of soluble silicon forms under excessive reactive oxygen species (ROS) and an upregulated immune response, as observed in biological fluids, cell lines, and animal models, raising the question of whether dietary intake of silicon and plasma silicon level influence systemic redox and inflammatory status in patients with RA.

Therefore, the aim of this study was to evaluate the silicon intake and plasma level in RA subjects and controls; moreover, the goal was to analyze the relationship of dietary and circulating silicon levels to selected serum indices of antioxidant, oxidant, and inflammatory status as measured in RA patients and compared to control subjects.

\section{Subjects and methods}

\section{Patients with rheumatoid arthritis and the controls}

This study included 115 RA patients who were enrolled in the study via the outpatient clinic of the Department of Rheumatology and Internal Medicine of Wroclaw Medical University; all of them fulfilled the American College of Rheumatology criteria. ${ }^{17}$ The control subjects comprised 129 healthy people recruited from public offices and Wrocław's $3^{\text {rd }}$ Age Universities. The exclusion criteria for the control group were chronic pro-inflammatory diseases and mental health issues. The study protocol was approved by the Wroclaw Medical University Ethics Board (consent No. KB-390/2012). All subjects gave written informed consent.

The RA study group underwent clinical examination by a rheumatologist in order to collect the following data: age, duration of disease, number of swollen joints, number of painful joints, the Disease Activity Score of 28 Joints (DAS 28), the presence of rheumatoid factor (RF) and anticitrullinated protein antibodies (ACPA), and pharmacological treatment. In both groups, body mass index (BMI) was measured and declared smoking status was recorded. In all recruited subjects, an assessment of dietary intake and habits was performed by a trained dietician, and fasting blood samples were drawn on the day of the dietary interview. Serum samples were collected in order to assess systemic redox and inflammatory status. To measure silicon levels, $\mathrm{K}_{2}$ EDTA plasma samples were collected with the exclusive use of plasticware in order to prevent preanalysis silicon contamination of the samples.

The 2 groups were matched in terms of age and cigarette smoking. The baseline characteristics of both groups are presented in Table 1. 
Table 1. Group characteristics

\begin{tabular}{|c|c|c|c|c|}
\hline \multicolumn{2}{|c|}{ Parameter } & $\begin{array}{c}\mathrm{RA} \\
(\mathrm{n}=115)\end{array}$ & $\begin{array}{l}\text { Control } \\
(\mathrm{n}=129)\end{array}$ & p-value \\
\hline \multirow{2}{*}{$\begin{array}{l}\text { Sex of subjects, } \\
\text { n (\%) }\end{array}$} & female & $91(79.1)$ & $82(63.6 \%)$ & \multirow{2}{*}{0.008} \\
\hline & male & $24(20.9)$ & $47(36.4 \%)$ & \\
\hline \multicolumn{2}{|c|}{ Age [years], median (range) Q1-Q3 } & $52(18-82) 43-63$ & $54(25-79) 46-65$ & NS \\
\hline \multirow{3}{*}{ BMI [kg/m²], n (\%) } & $<18.5$ & $2(1.7)$ & $3(2.3 \%)$ & \multirow{3}{*}{ NS } \\
\hline & $18.5-24.9$ & $48(41.7)$ & $44(34.1 \%)$ & \\
\hline & $\geq 25$ & $65(56.5)$ & $82(63.6 \%)$ & \\
\hline \multirow{2}{*}{$\begin{array}{l}\text { Cigarette smoking, } \\
\mathrm{n}(\%)\end{array}$} & current smoker & $33(28.7)$ & $41(31.8 \%)$ & \multirow{2}{*}{ NS } \\
\hline & former smoker (cessation $\geq 1$ year) \& never smoker & $82(71.3)$ & $88(68.2 \%)$ & \\
\hline \multicolumn{2}{|c|}{ Time from RA onset [years], median (range) } & $8.9(0.2-50)$ & - & - \\
\hline \multicolumn{2}{|c|}{ Number of swollen joints, median (range) } & $4(0-24)$ & - & - \\
\hline \multicolumn{2}{|c|}{ DAS 28, median (range) } & $5.05(1.49-8.52)$ & - & - \\
\hline \multicolumn{2}{|c|}{ RF+ (cutoff $20 \mathrm{IU} / \mathrm{mL}), \mathrm{n}(\%)$} & $56(65)$ & - & - \\
\hline \multicolumn{2}{|c|}{ ACPA+ (cutoff $25 \mathrm{U} / \mathrm{mL}), \mathrm{n}(\%)$} & $44(51)$ & - & - \\
\hline \multirow{4}{*}{$\begin{array}{l}\text { Treatment, } \\
\text { n (\%) }\end{array}$} & methotrexate & $46(56.1)$ & - & - \\
\hline & other non-biological DMARDs & $33(40.2)$ & - & - \\
\hline & anti-TNF therapy & $23(28.0)$ & - & - \\
\hline & steroids & $62(75.6)$ & - & - \\
\hline \multirow{11}{*}{$\begin{array}{l}\text { Dietary intakes, } \\
\text { median (range) }\end{array}$} & calories [kcal] & $1590.4(884.4-3622.5)$ & $1708.2(896.5-3816.2)$ & 0.037 \\
\hline & carbohydrates [g] & $225.5(112.3-373.9)$ & $233.3(92.5-588.4)$ & 0.019 \\
\hline & protein [g] & $65.8(28.4-145.7)$ & $69.0(31.8-174.5)$ & NS \\
\hline & fat $[g]$ & $50.1(20.6-136.7)$ & $56.5(15.5-148.2)$ & 0.022 \\
\hline & water $[g]$ & $2225.7(1057.9-3950.2)$ & $2035.9(717.5-5395.7)$ & NS \\
\hline & fiber $[g]$ & $18.0(6.8-34.6)$ & $20.9(6.0-48.3)$ & 0.007 \\
\hline & vitamin C [mg] & $83.6(18.8-459.5)$ & $93.0(11.9-505.3)$ & NS \\
\hline & vitamin E [mg a-tocopherol equiv.] & $6.10(1.89-83.21)$ & $7.13(2.75-19.21)$ & NS \\
\hline & vitamin A [ $\mu$ g retinol equiv.] & $756.3(292.4-10484.1)$ & 883.6 (210.8-9611.4) & NS \\
\hline & $\mathrm{Fe}[\mathrm{mg}]$ & $8.92(3.90-24.2)$ & $11.45(4.21-26.8)$ & 0.005 \\
\hline & $\mathrm{Cu}[\mathrm{mg}]$ & $0.90(0.49-6.81)$ & $1.24(0.55-2.45)$ & $<0.000$ \\
\hline
\end{tabular}

RA - rheumatoid arthritis patients; Control - control subjects; $\mathrm{n}$ - number of subjects; Q1-Q3 - range between the $25^{\text {th }}$ and $75^{\text {th }}$ percentile; BMI - body mass index; DAS 28 - Disease Activity Score of 28 joints; RF - rheumatoid factor; ACPA - anti-citrullinated protein antibody; DMARDs - disease-modifying anti-rheumatic drugs; anti-TNF - anti-tumor necrosis factor; NS - not statistically significant.

\section{Dietary intake assessment}

Dietary intake in the 2 groups was assessed by dietary recall from the last 3 days before the interview. ${ }^{18}$ Long-term silicon intake was assessed using a questionnaire on dietary habits developed by the Department of Food Science and Dietetics at the Wroclaw Medical University. ${ }^{19}$ The questionnaire consists of 106 questions concerning the consumption of food groups and individual foods within those groups, calculated in portions per unit of time. The amounts of dietary silicon were calculated using data from unpublished analyses of Polish food products which were performed at the Department of Food Science and Dietetics, as well as the United Kingdom silicon food database $^{4}$ and data on silicon contents in Belgian food products. ${ }^{3}$ As the dietary habit questionnaire has been validated in the Polish population only for the dietary intake of nutrients with established daily recommendation, but not for silicon, silicon intake from the 3-day dietary recall was also calculated to cross-check the data gathered from the dietary habit questionnaire. Silicon intake calculated using the 3 -day food recall amounted to $90 \pm 9 \%$ of the intake reported on in the dietary habit questionnaire. Moreover, the data from the dietary recall allowed us to assess short-term dietary exposure to silicon. As silicon intake and plasma levels may differ depending on gender, the 2 variables were measured in each group as a whole and in the female and male subgroups. ${ }^{6,20}$

\section{Silicon determination in plasma}

The levels of silicon in plasma were determined by graphite furnace-atomic absorption spectrometry (GF-AAS), using the standard addition calibration method. A PinAAcle 900 
(Perkin Elmer, Waltham, USA) atomic absorption spectrometer was used for analyses. All reagents were prepared using deionized water with a specific resistivity of $18.2 \mathrm{M} \Omega-\mathrm{cm}$. Plasma samples were diluted (1:4) with $0.01 \%$ Triton X-100 in water. From a silicon standard solution of $1000 \pm 3 \mathrm{mg} / \mathrm{L}$ in $0.2 \% \mathrm{HNO}_{3}$ (CPI International, Santa Rosa, USA), working standard solutions were prepared in 60 and $120 \mu \mathrm{g} / \mathrm{L}$ volumes. The graphite furnace was injected with $10 \mu \mathrm{L}$ of sample, $10 \mu \mathrm{L}$ of water or consecutive standard solution, and $5 \mu \mathrm{L}$ of matrix modifier $(0.1 \% \mathrm{Pd}$ and $0.01 \% \mathrm{Mg})$. The operating conditions and parameters of the instruments for silicon measurement are summarized in Table 2 . The silicon signal was linear up to $700 \mu \mathrm{g} / \mathrm{L}$ with the limit of quantification $5 \mu \mathrm{g} / \mathrm{L}$. Due to the lack of appropriate certified reference material, the Seronorm ${ }^{\mathrm{TM}}$ Trace Element Serum L-2 (Sero AS, Billingstad, Norway) was used with an approximate concentration of silicon given (ICP-SFMS), and the recovery rate was $106 \%$.

Table 2. Operating conditions and instrumental parameters for silicon measurement in plasma by graphite furnace - atomic absorption spectrometry (GF-AAS)

\begin{tabular}{|c|c|c|c|c|}
\hline $\begin{array}{l}\text { Operating } \\
\text { conditions }\end{array}$ & $\begin{array}{c}\text { Temperature } \\
{\left[{ }^{\circ} \mathrm{C}\right]}\end{array}$ & $\begin{array}{l}\text { Ramp } \\
\text { time }[\mathrm{s}]\end{array}$ & $\begin{array}{l}\text { Hold time } \\
\qquad[s]\end{array}$ & Gas \\
\hline Drying & $\begin{array}{l}110 \\
130 \\
300\end{array}$ & $\begin{array}{r}1 \\
30 \\
20\end{array}$ & $\begin{array}{r}5 \\
40 \\
5\end{array}$ & $\begin{array}{l}\text { argon } \\
\text { argon } \\
\text { argon }\end{array}$ \\
\hline Mineralization & $\begin{array}{l}550 \\
550\end{array}$ & $\begin{array}{r}20 \\
1\end{array}$ & $\begin{array}{l}15 \\
15\end{array}$ & $\begin{array}{c}\text { air } \\
\text { argon }\end{array}$ \\
\hline Pyrolysis & $\begin{array}{l}1100 \\
1250\end{array}$ & $\begin{array}{l}5 \\
1\end{array}$ & $\begin{array}{l}10 \\
30\end{array}$ & $\begin{array}{l}\text { argon } \\
\text { argon }\end{array}$ \\
\hline Atomization & 2450 & 0 & 5 & - \\
\hline Cleaning & 2550 & 1 & 3 & argon \\
\hline \multicolumn{5}{|c|}{ Instrumental parameters } \\
\hline \multicolumn{2}{|l|}{ Wavelength } & \multicolumn{3}{|c|}{$251.61 \mathrm{~nm}$} \\
\hline \multicolumn{2}{|l|}{ Slit width } & \multicolumn{3}{|c|}{$0.2 \mathrm{~nm}$} \\
\hline \multicolumn{2}{|l|}{ Lamp energy } & \multicolumn{3}{|c|}{$63 \mathrm{~mA}$} \\
\hline \multicolumn{2}{|c|}{ Measurement mode } & \multicolumn{3}{|c|}{ area under the peak } \\
\hline \multicolumn{2}{|c|}{ Characteristic mass } & \multicolumn{3}{|c|}{$42 \mathrm{pg}$} \\
\hline
\end{tabular}

\section{Biochemical analyses in serum}

The total antioxidant status (TAS) in the blood serum was measured by the spectrophotometric method with 2,2'-azino-bis(3-ethylbenzothiazoline-6-sulfonic acid) diammonium salt (ABTS), using a Randox TAS kit (Randox Laboratories, Crumlin, UK) and a Konelab 20i analyzer (ThermoSientific, USA). ${ }^{21}$ The 2,2'-diphenyl-1-picrylhydrazyl (DPPH) radical scavenging capacity was measured spectrophotometrically in deproteinized serum (DSAS) using a Spectronic GENESYS 6 UV-visible spectrophotometer (Thermo Electron Corporation, Waltham, USA). ${ }^{22}$ In both methods, antioxidant status was expressed in Trolox equivalents.
The total oxidant status (TOS) of the serum was measured as described by Erel. ${ }^{23}$ In this method, the ability of serum oxidants to oxidize ferrous-ion-o-dianisidine complex is ascertained by the spectrophotometric determination of colored oxidized product using xylenol orange. The assay was calibrated with hydrogen peroxide. The results are expressed in $\mu \mathrm{M}$ of $\mathrm{H}_{2} \mathrm{O}_{2}$ equivalent. Oxidative stress index (OSI) was also calculated as a TOS:TAS ratio, using the values of both parameters expressed in the given units. $^{24}$

Thiobarbituric acid reactive substances (TBARS) in the serum were detected spectrophotometrically after extracting pink-colored reaction products in butanol, and the TBARS concentration was expressed in nM of malondialdehyde (MDA). ${ }^{25}$

Serum albumin concentration was measured using an assay kit (Thermo Electron Corporation, Vantaa, Finland) with bromocresol green based on the colorimetric method. Uric acid was measured in the serum using a method based on oxidation by uricase. Serum high sensitivity C-reactive protein (hs-CRP) was determined using an hs-CRP assay kit (DiaSys, Holzheim, Germany) based on the measurement of immunoprecipitation at $540 \mathrm{~nm}$. The addition of microparticles coated with anti-human CRP to the buffered samples results in immunoprecipitation recorded turbidimetrically at its end-point. Albumin, uric acid, and hs-CRP were measured with the Konelab 20i analyser.

The activity of superoxide dismutase (SOD) in serum was measured with an SOD Activity Assay kit (Cayman Chemical, Ann Arbor, USA), which uses tetrazolium salt to quantify superoxide radicals generated by xanthine oxidase and hypoxanthine. The oxidation rate of tetrazolium salt to formazan dye is inversely proportional to the endogenous activity of SOD. One unit (U) of SOD activity was defined as the amount of enzyme causing $50 \%$ superoxide radical dismutation. The activity of catalase (CAT) was measured in the serum using a CAT Assay kit (Cayman Chemical) which uses the peroxidative function of the enzyme at optimal hydrogen peroxide concentration with methanol as a substrate. One unit (U) of CAT activity was defined as the amount of enzyme causing the formation of $1 \mathrm{nmol}$ of formaldehyde per minute. Measurements of SOD and CAT were performed on a Multiscan GO microplate reader (Thermo Fisher Scientific, Waltham, USA).

Serum interleukin-6 (IL-6) concentration was also measured, using a solid phase sandwich ELISA assay kit (Diaclone SAS, Besançon, France).

\section{Statistical analyses}

Statistical calculations were performed using STATISTICA v. 13.0 (StatSoft Inc., Tulsa, USA). Pearson's $\chi^{2}$ test was used to assess differences in the qualitative variables between groups. The correlations between measured variables were assessed in both groups, further divided into 
subgroups by gender, smoking status, and current treatment. Depending on the distribution of variables, either Student's t-test or the Mann-Whitney U test were used for group comparisons, and either Pearson or Spearman correlation analysis was used to determine associations between variables.

\section{Results}

Data on the intake of macronutrients, antioxidant vitamins, and essential microelements involved in peroxyl radical formation is included in Table 1 . The diet of the experimental group provided the subjects with less energy, fat, carbohydrates, dietary fiber, iron, and copper than that of the control group, though their intakes of vitamin $C$ and equivalents of vitamins E and A were similar. Short-term silicon intake estimated from the 3-day dietary recall amounted to $21.4 \mathrm{mg} /$ day in the experimental group and was approx. $10 \%$ higher than in the control group; this difference was not observed in men, however (Table 3). The main dietary sources of silicon for the 2 study groups were cereal products (approx. $35 \%$ in total) and hot and cold non-alcoholic beverages (34 and 24\% in total, respectively). Among cereal products, breads played an important role in the acquisition of dietary silicon. Non-alcoholic beverages contributed significantly more to silicon intake in the RA group than in the controls. Fruits and vegetables also provided considerable amounts of silicon in the diets of both groups, whereas fruits comprised a more substantial source for the controls.

Plasma concentrations of Si were shown to vary over a wide range in both groups, but the interquartile range between the $25^{\text {th }}$ and $75^{\text {th }}$ percentile was smaller in the controls than in the experimental group, including the distribution of values in the subgroups of females and males (Table 3). Significantly higher concentrations of silicon were found in the RA group than in the control group, and especially large differences were observed between the females of these groups; the males' plasma silicon concentration did not differ significantly in either group. In the controls, a negative correlation between silicon concentration and age was also noticed. A negative correlation between silicon level and the number of swollen joints was found in the RA female subgroup.

Total silicon intake estimated according to the short-term dietary recall did not correlate with silicon concentration in plasma, while dietary silicon provided by non-alcoholic beverages was shown to correlate with plasma silicon in the RA group. In the control group, the total silicon intake from cereal products correlated with plasma silicon level.

Table 3. Silicon intake and plasma level in the RA and control groups, and significant correlations found between plasma or dietary silicon and selected characteristics in the study groups

\begin{tabular}{|c|c|c|c|c|}
\hline \multicolumn{2}{|c|}{ Parameter } & RA & Control & $\mathrm{p}$-value \\
\hline \multirow{3}{*}{$\begin{array}{l}\text { Silicon intake }[\mathrm{mg} / \text { day], } \\
\text { median (range) }\end{array}$} & all subjects & $21.4(11.2-48.6)$ & $19.4(12.6-37.0)$ & 0.046 \\
\hline & female & $24.4(12.9-48.6)$ & $19.9(12.6-37.0)$ & 0.041 \\
\hline & male & $20.1(11.2-31.1)$ & $19.8(15.0-30.6)$ & NS \\
\hline \multirow{9}{*}{$\begin{array}{l}\text { Silicon food sources } \\
\text { [\% of total silicon intake] }\end{array}$} & white bread & 13.0 & 13.1 & NS \\
\hline & wholemeal bread & 9.6 & 9.3 & NS \\
\hline & other cereal products & 12.5 & 13.5 & NS \\
\hline & vegetables & 11.3 & 13.2 & NS \\
\hline & fruits & 9.1 & 14.1 & 0.003 \\
\hline & non-alcoholic beverages & 19.9 & 11.3 & $<0.000$ \\
\hline & tea \& coffee & 13.8 & 12.6 & 0.037 \\
\hline & alcoholic beverages & 0.5 & 3.3 & 0.007 \\
\hline & other foods & 10.3 & 9.6 & NS \\
\hline \multirow{3}{*}{$\begin{array}{l}\text { Plasma silicon }[\mu \mathrm{g} / \mathrm{L}] \\
\text { median (range) } \mathrm{Q} 1-\mathrm{Q} 3\end{array}$} & all subjects & $\begin{array}{c}192.3(42.7-372.9) \\
120.2-269.7\end{array}$ & $\begin{array}{c}140.9(59.4-312.0) \\
111.0-175.9\end{array}$ & 0.028 \\
\hline & female & $\begin{array}{c}196.6(42.7-372.9) \\
118.7-269.1\end{array}$ & $\begin{array}{c}139.1(66.1-312.0) \\
104.2-177.2\end{array}$ & 0.007 \\
\hline & male & $\begin{array}{c}207.7(44.6-366.1) \\
131.9-279.7\end{array}$ & $\begin{array}{c}149.2(59.4-269.8) \\
115.5-170.4\end{array}$ & NS \\
\hline \multirow{3}{*}{$\begin{array}{l}\text { Significant plasma silicon correlations } \\
\text { with food consumption }(r)\end{array}$} & cereal products & - & 0.52 & 0.001 \\
\hline & tea \& coffee & 0.33 & - & $<0.000$ \\
\hline & non-alcoholic cold beverages & 0.29 & - & 0.002 \\
\hline \multirow{2}{*}{$\begin{array}{l}\text { Significant plasma silicon correlations } \\
\text { with group characteristics }(r)\end{array}$} & age & - & -0.27 & 0.001 \\
\hline & number of swollen joints & -0.32 & - & 0.041 \\
\hline
\end{tabular}

RA - rheumatoid arthritis patients; Control - control subjects; Q1-Q3 - range between the $25^{\text {th }}$ and $75^{\text {th }}$ percentiles; NS - not statistically significant; $r$ - correlation coefficient. 
Table 4. Redox status components and markers of inflammation in RA and control groups, median (range)

\begin{tabular}{|l|c|c|c|}
\multicolumn{1}{|c|}{ Parameter } & RA & Control & p-value \\
\hline TAS $[\mathrm{mM}$ Trolox] & $1.47(1.03-1.86)$ & $1.71(1.20-2.14)$ & $<0.000$ \\
\hline DSAS $[\mu \mathrm{M}$ Trolox] & $171.3(41.1-450.3)$ & $202.6(67.4-523.1)$ & $<0.000$ \\
\hline TOS $\left[\mu \mathrm{M} \mathrm{H} \mathrm{O}_{2}\right.$ equiv. & $7.81(2.25-14.94)$ & $2.84(0.09-49.31)$ & $<0.000$ \\
\hline OSI (TOS/TAS ratio) & $5.19(1.76-10.03)$ & $1.63(0.05-32.96)$ & $<0.000$ \\
\hline TBARS $[\mu \mathrm{M} \mathrm{MDA}]$ & $1.88(1.16-4.04)$ & $1.75(0.68-7.98)$ & 0.001 \\
\hline SOD $[\mathrm{U} / \mathrm{mL}]$ & $2.49(0.32-4.57)$ & $1.95(0.19-3.22)$ & 0.031 \\
\hline CAT $[\mathrm{U} / \mathrm{mL}]$ & $35.63(4.70-76.24)$ & $40.27(5.48-77.91)$ & 0.023 \\
\hline Albumin $[\mathrm{g} / \mathrm{dL}]$ & $3.92(2.52-5.7)$ & $4.25(2.85-5.42)$ & 0.006 \\
\hline Uric acid $[\mathrm{g} / \mathrm{dL}]$ & $4.4(1.5-9.1)$ & $5.3(3.0-8.8)$ & NS \\
\hline IL-6 $[\mathrm{pg} / \mathrm{mL}]$ & $6.39(0.84-98.95)$ & $2.26(0.81-16.41)$ & $<0.000$ \\
\hline hs-CRP $[\mathrm{mg} / \mathrm{L}]$ & $4.94(0.12-83.57)$ & $1.51(0.03-10.8)$ & $<0.000$ \\
\hline
\end{tabular}

RA - rheumatoid arthritis patients; Control - control subjects; TAS - total antioxidant status; DSAS - deproteinized serum antioxidant status; TOS - total oxidant status; OSI - oxidative status index; TBARS - thiobarbituric acid reactive substances; SOD - superoxide dismutase; CAT - catalase; IL-6 - interleukin 6; hs-CRP - high sensitivity C-reactive protein; NS - not statistically significant.

Table 5. Correlations between dietary or plasma silicon with markers of redox and inflammatory status in RA and control groups

\begin{tabular}{|l|c|c|c|c|}
\multirow{2}{*}{ Parameter } & \multicolumn{2}{|c|}{ Correlations with dietary Si } & \multicolumn{2}{c|}{ Correlations with plasma Si } \\
\cline { 2 - 5 } & RA & Control & RA & Control \\
\hline TAS & NS & NS & $0.34(0.007)^{\mathrm{a}}$ & $0.28(0.001)$ \\
\hline DSAS & NS & NS & $-0.23(0.018)$ & $0.25(0.014)$ \\
\hline TOS & $-0.28(0.009)^{\mathrm{c}}$ & NS & $-0.24(0.032)^{\mathrm{b}}$ & $-0.23(0.007)$ \\
\hline OSI & $-0.26(0.014)^{\mathrm{c}}$ & NS & $-0.30(0.019)^{\mathrm{a}}$ & $-0.26(0.003)$ \\
\hline TBARS & NS & NS & NS & $0.34(0.017)^{\mathrm{d}}$ \\
\hline Albumin & $0.28(0.030)^{\mathrm{a}}$ & NS & NS & NS \\
\hline Uric acid & NS & $-0.42(0.005)^{\mathrm{b}}$ & NS & NS \\
\hline SOD & NS & NS & NS & NS \\
\hline CAT & NS & NS & $0.21(0.027)$ & NS \\
\hline hs-CRP & $-0.35(0.012)^{\mathrm{a}}$ & NS & NS & NS \\
\hline IL-6 & $-0.19(0.041)$ & NS & $-0.32(0.005)^{\mathrm{b}}$ & NS \\
\hline
\end{tabular}

RA - rheumatoid arthritis patients; Control - control subjects; TAS - total antioxidant status; DSAS - deproteinized serum antioxidant status; TOS - total oxidant status; OSI - oxidative status index; TBARS - thiobarbituric acid reactive substances; SOD - superoxide dismutase; CAT - catalase; IL-6 - interleukin 6; hs-CRP - high sensitivity C-reactive protein; NS - not statistically significant. Statistical significance in subgroups: ${ }^{\text {a }}$ - patients with an albumin level above the median value $(3.92 \mathrm{~g} / \mathrm{dL}) ;{ }^{b}$ - non-smokers; ${ }^{c}$ - female subjects; ${ }^{\mathrm{d}}$ - male subjects.

For the assessment of systemic redox balance in the study groups, the antioxidant capacity and total oxidant load in serum were measured (Table 4). The scavenging activity of non-enzymatic serum components against ABTS radicals was determined by TAS assay. The antiradical activity of low-molecular-weight serum components was also assessed in deproteinized serum using a DPPH-stable radical (DSAS assay). Significantly lower values of both TAS and DSAS measurements were found in the RA group than in the controls. In both groups, DSAS values comprised approx. $12 \%$ of the TAS. Measuring the concentrations of the main endogenous antioxidants in blood serum revealed that in the experimental group circulated albumins were lower than in the control group; in the case of uric acid, no differences were found. The total concentration of oxidant molecules in blood serum was measured by TOS assay, which revealed higher values among the RA group than the control group. These differences in oxidative status between groups were accompanied by higher OSI values for RA patients than for controls. The TBARS assay used in this study as an indicator of lipid peroxidation also showed higher serum concentrations of MDA equivalents in the experimental group. The assessment of the enzymatic components of antioxidant status revealed more activity of serum SOD and less activity of CAT in the RA group than in the control group. Disrupted systemic inflammatory status, expressed as a higher serum concentration of hs-CRP and IL-6, was also found in the RA patients than in the controls (Table 4).

The relationships of dietary intake and plasma silicon concentration to redox and inflammatory statuses were statistically analyzed for the 2 groups (Table 5). No correlation between plasma silicon and dietary silicon level was observed in either group, though a negative correlation between $\mathrm{Si}$ intake and IL-6 level was found among the RA group, and negative correlations of $\mathrm{Si}$ intake with TOS and OSI was noted among the RA females, as well as with hs-CRP in patients with above-average serum albumin levels. Moreover, silicon intake in the RA group was associated with higher concentrations of albumin; however, in patients with albumin levels above the median, this relationship was not noticed. In the control group, dietary silicon did not correlate with redox or inflammatory status, except for a negative correlation with uric acid. Plasma silicon concentration positively correlated with TAS and DSAS values in the controls. In the experimental group, a positive correlation of plasma silicon with TAS was found in patients with serum albumin over the median value, and this relationship was accompanied by an opposite relationship with DSAS and was observed in all RA patients. Among the parameters of oxidative status, TOS and OSI negatively correlated with plasma silicon in the experimental group. In the case of TOS, this correlation was limited to non-smoking patients; for OSI it was limited to patients with above-average albumin levels. In the control group, these 2 parameters decreased with increasing plasma silicon concentrations. A positive correlation between TBARS level and plasma 
silicon level was noticed, but only among the male control subjects. The activity of SOD and CAT did not correlate to plasma silicon in the controls, while a positive correlation between plasma silicon and CAT was found among the RA group, especially in those with above-average albumin levels. Plasma silicon concentration did not correlate with inflammatory markers in the control group. In the experimental group, a negative correlation with IL-6 concentration was found, but only in non-smoking patients and in the female subgroup.

\section{Discussion}

This study is the first attempt to assess the association of silicon provided by diet and silicon concentration in blood plasma with the antioxidant and oxidant status of patients with RA and with systemic markers of inflammatory status in RA. Our previous study showed that the intake of some essential nutrients, such as polyunsaturated fatty acids, vitamin E, B6, and calcium correlated with higher serum antiradical activity in RA patients; moreover, this antioxidant potential was beneficially linked with disease duration and severity. ${ }^{18}$ Silicon also seems to be involved in redox balance and inflammation, though these observations originate from animal models and the cell cultures underwent toxic, metabolic, or immune challenges. ${ }^{7-10}$ The effects of dietary silicon on the development, progress, and severity of autoimmune diseases have not yet been studied.

Dietary Si intake has not yet been evaluated in the Polish population and no database is available with silicon levels in Polish foods. Therefore, we assessed the amount of silicon in foods consumed by the subjects in this study by using the dietary recall method and a questionnaire on dietary habits, and by calculating silicon intake according to data on the silicon content of foods measured in our laboratory and databases on food component analysis available in European countries and published elsewhere. ${ }^{3,4}$ So far, adequate daily intakes of Si have not been established, but the results obtained in this study are in agreement with the estimated beneficial intake range based on animal and human exposure studies (10-30 mg/day); according to the Framingham Offspring Cohort study, however, the most favorable amount of silicon in the diet associated with bone health exceeded $30 \mathrm{mg} /$ day. ${ }^{1,26}$

The contributions of foods supplying silicon in the 2 study groups were in alignment with previous studies, though the higher intake in male subjects than female subjects was not confirmed. ${ }^{3}$ As with Finnish, British, and American diets, cereal products were the primary food source of silicon, followed by fruits and vegetables and hot and cold beverages. In the typical Western diet, beer was among the foods found to contribute the most to the intake of silicon in men (up to $18 \%$ ). ${ }^{26}$ In our study, alcoholic beverages proved to be a much less important source of $\mathrm{Si}$ in the diet. The RA group, especially women, consumed a diet providing slightly more silicon than the control group, and non-alcoholic beverages containing soluble orthosilicic acid comprised a larger percentage of silicon food sources for the RA patients than the controls. Silicon sources in the diets of both groups greatly reflected the dietary habits of the adult Polish population, as recently published in the National MultiCenter Health Survey (WOBASZ II, 2013-14), ${ }^{27,28}$ and in our previous study concerning the diet quality of RA patients vs controls. ${ }^{18}$ These habits include consuming a high proportion of white bread to total cereal products, tea and coffee comprising much of the non-alcoholic beverages, and fruit and vegetable consumption which is lower than recommended.

A higher silicon content in the diet was accompanied by a higher plasma silicon level in the RA group than in the control group. A positive correlation of plasma silicon with beverage consumption has also been found in RA patients, which provides an easily absorbable form of silicon. ${ }^{3}$ Reference values for fasting plasma silicon concentration in healthy adults have not yet been established. It is difficult to refer measured plasma silicon levels to the data published for different populations because of the different methods used in measuring silicon concentration and the lack of certified reference materials. Nevertheless, it may be stated that the concentrations of silicon in the plasma of the subjects in this study were similar to those obtained for other populations (100-310 $\mu \mathrm{g} / \mathrm{L}){ }^{1}{ }^{1}$ The negative correlation with age found among the controls was in alignment with previous findings, ${ }^{3}$ while in the RA patients no correlation between plasma silicon and age was noted, and in the female RA subgroup a favorable association with the disease course was found. Plasma silicon concentration did not appear to be dependent on gender, as observed by other authors. ${ }^{24}$ Since it has been shown that plasma silicon level is physiologically regulated by kidney excretion and likely by active intestine transport, the elevated plasma silicon levels among the RA patients may suggest an alteration in this regulation linked to the disease rather than a slightly higher oral intake of silicon in these patients. ${ }^{1,2}$ An increased serum silicon level has been reported in rats with collagen-induced arthritis, and higher serum Si levels have also been observed in sclerosis multiplex - a disease with inflammatory immunopathogenesis. ${ }^{8,29}$

The role of oxidative stress has been implicated in the etiology of RA, and several studies have observed a relationship between oxidative damage and the maintenance of the inflammatory process in RA. ${ }^{15}$ In this study, we assessed extracellular antioxidant/oxidant status, since it constitutes an important modulator of intracellular redox balance in different tissues and under inflammatory conditions. ${ }^{30}$ Systemic antioxidant status was analyzed using TAS and DSAS methods which evaluate serum radical scavenging capacity, being the first line of defense in conditions of increased ROS production. 
In order to specify the potential of the circulating lowmolecular-weight antioxidant pool, measurements of antiradical activity in deproteinized serum (DSAS) were taken in addition to TAS. ${ }^{31}$ As anticipated, the RA experimental group was characterized by an imbalance in redox status, manifested in the reduced antiradical capacity of serum and its deproteinized fraction. Elevated serum TOS, OSI, and TBARS levels in comparison with the control group also indicated an overproduction of hydrogen peroxides and lipid peroxides. ${ }^{23}$ The disrupted balance between SOD and CAT activity that was found in the RA group when compared to the control group may contribute considerably to the impaired management of hydrogen peroxide production. Activity of $\mathrm{Cu} / \mathrm{Zn}-\mathrm{SOD}$ has previously been shown to be elevated or reduced in the serum or plasma of RA patients. ${ }^{16,32}$ The inhibition of serum CAT activity observed in the RA group was in accordance with Shah et al., 33 though other authors have found this enzyme to be more active. ${ }^{32}$ Insufficiently metabolized hydrogen peroxide, under reduced CAT activity, underlies further reactions to yield the highly reactive hydroxyl radical involved in lipid oxidation, and might therefore contribute to the increased formation of MDA found in the TBARS assay of the RA group. The lower serum concentrations of albumins in the RA group when compared to the control group indicate diminished cationic ligand binding by albumins, thereby facilitating the formation of hydroxyl radicals via the Fenton reaction. ${ }^{31,34}$

The inflammatory response in RA involves the increased expression of markers of inflammation, such as CRP and proinflammatory cytokines, including tumor necrosis factor- $\alpha$ (TNF- $\alpha$ ) and IL- 6 , in the synovial fluid and serum. ${ }^{35}$ In our study, both hs-CRP and IL- 6 concentrations were significantly higher in the serum of RA patients than in the controls. Concentrations of IL- 6 higher than those in the control group were also found for patients treated with anti-TNF therapy (data not shown). Interleukin 6 plays a crucial role in RA pathogenesis and may serve as an indicator of disease severity. ${ }^{36}$ The immense value of CRP as an inflammatory marker in RA has also been recognized. $C$ reactive protein, as part of an acute phase response to inflammation, stimulates proinflammatory cytokine production and may contribute directly to the inflammatory state. ${ }^{15,35}$

No link between dietary silicon and indices of oxidant/ antioxidant status was found in the control subjects, except for a negative correlation with serum uric acid concentration. The effect of silicon on uric acid level has not yet been reported; however, the relationship noted in this study may be indirect, since dietary silicon sources (such as cereals, fruits, and vegetables) are simultaneously purine-poor foods, leading to a decrease of uric acid level in the serum. ${ }^{37}$ The total Si intake from these foods was higher in the control group than in the experimental group (63.2\% vs $55.5 \%$, respectively). In the conditions of enhanced oxidative stress detected in the RA patients, the correlation between dietary silicon and serum markers of redox status was more pronounced and pertained to the reduction of hydrogen peroxide and lipid peroxide levels measured in the TOS assay for female patients with increased silicon content in their diet. Moreover, the ratio between oxidant and antioxidant status decreased along with increasing silicon intake in these subjects, indicating the role of $\mathrm{Si}$ in managing oxidant overproduction related to RA. Again, the observed relationship could be an effect of the composition of foods supplying silicon, which are mainly of plant origin and contain a wealth of antioxidants proven to be effective in ROS neutralization. However, it has been shown that silicon possesses the ability to remove hydrogen peroxide or other ROS, and its protective effects against hydrogen peroxide toxicity was observed in animal models and cell lines. ${ }^{2,9,38}$ Moreover, it has been reported that silicon exerts an in vitro inhibitory effect on DPPH radical production in a concentration-dependent manner. ${ }^{9}$ This specific implication of silicon in managing oxidative stress was confirmed in our study by the positive correlation between silicon concentration in plasma and antiradical capacity measures, and by the negative correlation to oxidative stress indices prominently expressed among the controls. In the RA patients, these correlations were found only in patients who were not burdened by factors influencing redox status, i.e., lower albumin levels and cigarette smoking. Uric acid contributes the most to the antioxidant pool of deproteinized serum, and the opposite relationship of silicon concentration to antiradical activity measured in deproteinized serum might result from the silicon-uric acid interactions revealed when silicon levels are elevated in the blood. Uric acid antioxidant activity has been shown in fact to be affected by certain components of the chemical milieu in the human body, including bicarbonate. ${ }^{39}$ Moreover, in the DPPH assay, which was performed in an organic solvent, the mode of interaction between the assay components and the impact of their proportions may differ from how they are in the natural environment. ${ }^{40}$ The compelling positive link between plasma silicon level and the marker of lipid peroxidation in male controls might be at least partially explained by the findings published by Garcimartin et al. ${ }^{38}$ The authors of that study demonstrated that silicon in concentrations up to $100 \mu \mathrm{g} / \mathrm{L}$ did not induce TBARS production in neuroblastoma cells, but when concentrations reached $250 \mu \mathrm{g} / \mathrm{L}$ or more peroxidation was significantly higher. This suggests that $\mathrm{Si}$ is ineffective as a hydroxyl radical neutralizer or may even induce the production of hydroxyl radicals at the concentrations found in serum. However, in this study an organic compound of silicon was tested, and the observed effect might depend on the chemical form of silicon. On the other hand, in an aluminum challenge study on rats, the concentration of TBARS decreased after oral administration of orthosilic acid, but this effect might result from the ability of silicon to produce aluminosilicate 
complexes rather than from direct antioxidant action. ${ }^{41}$ It should also be mentioned that a high intake of iron and copper was noted among the control group, especially in the men, whose diet supplied $135 \%$ and $153 \%$ of the recommended daily allowance for these elements, respectively. ${ }^{42}$ Moreover, a tendency toward higher plasma silicon levels was observed in the male controls with higher Fe intake (data not shown). An excessive intake of these redox-active transition metals, especially iron, has been shown to promote lipid peroxidation, so this effect might contribute to the relationship between plasma silicon level and TBARS level observed in the male subgroup. ${ }^{43}$ Further light on the role of silicon in extracellular redox status may be shed by the relationship of Si to antioxidant enzyme activity in the serum. Although dietary and plasma silicon were not linked to SOD and CAT activity in serum among the control group, a significant positive correlation was found for CAT in the RA patients, especially among those with above-average albumin values. In RA, silicon may play a modulatory role in ROS disabling, where SOD activity is elevated and CAT is reduced. These associations suggest that silicon in the serum acts as a hydrogen peroxide neutralizer, at least partly by inducing CAT activity. The modulatory effects of silicon on SOD and CAT were also studied in a non-alcoholic steatohepatitis rat model, where strong activation of initially low SOD levels in the liver tissue of rats was reported after the rats were fed supplemental silicon, while the activities of CAT and glutathione peroxidase did not change. ${ }^{10}$ As the authors of that study suggested, in this model the ability of silicon to neutralize hydrogen peroxide rendered the overexpression of CAT unnecessary. A favorable link between dietary and plasma Si and inflammatory response in RA was also found in our study, but some interferences were noted - given that this relationship was influenced by serum albumin level, smoking status, or gender. Rheumatoid arthritis therapy did not further modify the observed associations (data not shown).

The role of silicon in alleviating inflammatory and immune response was revealed in a study on animals with collagen-induced arthritis. ${ }^{8}$ The involvement of silicon in the response to inflammation was also demonstrated in silicon-deprived rats, which accumulate more silicon in the liver and bones than silicon-supplemented rats in acute-phase inflammation induced by lipopolysaccharide endotoxin. ${ }^{44}$ In murine macrophage cells, the inhibitory effect of silicon on IL- 6 secretion was observed only at a concentration of $50 \mu \mathrm{M}$, far exceeding those found in human blood. ${ }^{9}$ Silicon was also shown to normalize the gene expression of TNF- $\alpha$ upregulated in mice exposed to aluminum. ${ }^{45}$ Since both hs-CRP and IL- 6 concentrations in the blood are sensitive markers for assessing the intensity of inflammatory processes and disease severity, the results obtained in this study may suggest a beneficial role of silicon in the course of RA. Bone resorption and osteoclast induction in RA is stimulated by IL-6, so the negative correlation between Si and IL- 6 in the RA group is an indication of its role in preventing bone, cartilage, and joint destruction in RA. ${ }^{35}$

In our previous study, systemic antioxidant capacity was shown to decrease in RA patients, as disease activity and lenght of time from disease onset increased. ${ }^{18} \mathrm{With}$ regard to this observation and the suggested contribution of silicon to antioxidant status in RA, we evaluated the association of dietary and plasma silicon with the clinical indices used to monitor RA activity and duration. A negative correlation between plasma silicon and the number of swollen joints was revealed among female RA patients; moreover, a non-significant positive trend was also noted for disease duration among non-smoking subjects $(r=0.19, \mathrm{p}=0.068)$. These findings may support the case for silicon's involvement in disease response in RA patients, though the effect of supplementary silicon on the number of affected joints was not found in an arthritic rat model. ${ }^{8}$

In conclusion, silicon may contribute to systemic redox balance. In light of the generally favorable correlation of plasma and dietary silicon to redox and inflammatory markers, the elevated plasma silicon levels in the RA patients (as compared to the controls) might suggest the involvement of silicon in managing the disturbances related to RA. Non-smoking status and non-affected serum albumin seem to be important in exposing the beneficial effects of silicon in RA. As a number of questions remain, further studies on the role of silicon in the antioxidant system and inflammatory processes are warranted.

\section{ORCID iDs}

Anna Prescha (D) https://orcid.org/0000-0002-4620-8953

Katarzyna Agnieszka Zabłocka-Słowińska

(D) https://orcid.org/0000-0003-2313-5728

Sylwia Płaczkowska (1) https://orcid.org/0000-0002-1466-3820

Daiva Gorczyca (D) https://orcid.org/0000-0002-5044-5513

Anna Łuczak (D) https://orcid.org/0000-0003-1934-6039

Halina Grajeta (D) https://orcid.org/0000-0003-0150-5134

\section{References}

1. Nielsen $\mathrm{FH}$. Update on the possible nutritional importance of silicon. J Trace Elem Med Biol. 2014;28(4):379-382.

2. Ratcliffe $S$, Jugdaohsingh R, Vivancos J, et al. Identification of a mammalian silicon transporter. Am J Physiol Cell Physiol. 2017;312(5): C550-C561.

3. Robberecht H, Van Cauwenbergh R, Van Vlaslaer V, Hermans N. Dietary silicon intake in Belgium: Sources, availability from foods, and human serum levels. Sci Total Environ. 2009;407:4777-4782.

4. Powell JJ, McNaughton SA, Jugdaohsingh, et al. A provisional database for the silicon content of foods in the United Kingdom. Br J Nutr. 2005;94(5):804-812.

5. Prescha A, Zabłocka-Słowińska K, Hojka A, Grajeta H. Instant food products as a source of silicon. Food Chem. 2012;135(3):1756-1761.

6. Jugdaohsingh R. Silicon and bone health. J Nutr Health Aging 2007; 11(2):99-110.

7. Seaborn CD, Briske-Anderson M, Nielsen FH. An interaction between dietary silicon and arginine affects immune function indicated by conA-induced DNA synthesis of rat splenic T-lymphocytes. Biol Trace Elem Res. 2002;87(1-3):133-142.

8. Nielsen FH. A novel silicon complex is as effective as sodium metasilicate in enhancing the collagen-induced inflammatory response of silicon-deprived rats. J Trace Elem Med Biol. 2008;22(1):39-49. 
9. Kim EJ, Bu SY, Sung MK, et al. Analysis of antioxidant and anti-inflammatory activity of silicon in murine macrophages. Biol Trace Elem Res. 2013;156(1-3):329-337.

10. Santos-López JA, Garcimartín A, Merino P, et al. Effects of silicon vs hydroxytyrosol-enriched restructured pork on liver oxidation status of aged rats fed high-saturated/high-cholesterol diets. PloS One. 2016;11(1):e0147469. doi.org/10.1371/journal.pone.0147469

11. Khoshgoftarmanesh AH, Khodarahmi S, Haghighi M. Effect of silicon nutrition on lipid peroxidation and antioxidant response of cucumber plants exposed to salinity stress. Arch Agron Soil Sci. 2014;60(5): 639-653.

12. Najda J, Goss M, Gmínski J, et al. The antioxidant enzymes activity in the conditions of systemic hypersilicemia. Biol Trace Elem Res. 1994; 42(1):63-70.

13. Stolt $P$, Källberg $H$, Lundberg I, et al. Silica exposure is associated with increased risk of developing rheumatoid arthritis: Results from the Swedish EIRA study. Ann Rheum Dis. 2005;64:582-586.

14. Watad A, Rosenberg V, Tiosano S, et al. Silicone breast implants and the risk of autoimmune diseases: Real world analysis. Ann Rheum Dis. 2018;SAT0687:1191-1192.

15. Filippin LI, Vercelino R, Marroni NP, Xavier RM. Redox signalling and the inflammatory response in rheumatoid arthritis. Clin Exp Immunol. 2008;152(3):415-422.

16. Bae SC, Kim SJ, Sung MK. Inadequate antioxidant nutrient intake and altered plasma antioxidant status of rheumatoid arthritis patients. J Am Coll Nutr. 2003;22(4):311-315.

17. Aletaha $D$, Neogi $T$, Silman AJ, et al. Rheumatoid arthritis classification criteria: an American College of Rheumatology/European League Against Rheumatism Collaborative Initiative. Arthritis Rheum. 2010; 62:2569-2581.

18. Prescha A, Zabłocka-Słowińska K, Płaczkowska S, et al. Diet quality and its relationship with antioxidant status in patients with rheumatoid arthritis. Oxid Med Cell Longev, 2018; article ID 8506343. doi.org/ $10.1155 / 2018 / 8506343$

19. Ilow R, Królicka O, Regulska-Ilow B, Pluta J. Validation of a food frequency questionnaire for dietary intake estimation among students from Wroclaw. Bromat Chem Toksykol. 2005;38(3):313-320.

20. Bissé $E$, Epting $T$, Beil $A$, et al. Reference values for serum silicon in adults. Anal Biochem. 2005;337:130-135.

21. Erel O. A novel automated direct measurement method for total antioxidant capacity using a new generation, more stable ABTS radical cation. Clin Bioch. 2004;37(4):277-285.

22. Chrzczanowicz J, Gawron A, Zwolinska A, et al. Simple method for determining human serum 2,2-diphenyl-1-picryl-hydrazyl (DPPH) radical scavenging activity-possible application in clinical studies on dietary antioxidants. Clin Chem Lab Med. 2008:46(3):342-349.

23. Erel O. A new automated colorimetric method for measuring total oxidant status. Clin Biochem. 2005;38:1103-1111.

24. Harma M, Erel O. Increased oxidative stress in patients with hydatidiform mole. Swiss Med Week. 2003;133:563-566.

25. Rice-Evans C, Diplock AT, Symons MCR. Techniques in free radical research. Amsterdam, Elsevier Science Publishers BV, 1991.

26. Jugdaohsingh $\mathrm{R}$, Tucker $\mathrm{KL}$, Qiao N, et al. Dietary silicon intake is positively associated with bone mineral density in men and pre-menopausal women of the Framingham Offspring Cohort. JBone Miner Res. 2004;19:297-307.

27. Waśkiewicz A, Szcześniewska D, Szostak-Węgierek D, et al. Are dietary habits of the Polish population consistent with the recommendations for prevention of cardiovascular disease? — WOBASZ II project. Kardiol Pol. 2016;74(9):969-977.
28. Micek A, Grosso G, Polak M, et al. Association between tea and coffee consumption and prevalence of metabolic syndrome in Poland - results from the WOBASZ II study (2013-2014). Int J Food Sci Nutr. 2018;69(3):358-368.

29. Alimonti A, Ristori G, Giubilei F, et al. Serum chemical elements and oxidative status in Alzheimer's disease, Parkinson disease and multiple sclerosis. Neurotoxicology. 2007;28:450-456.

30. Jones IV AR, Meshulam T, Oliveira MF, et al. Extracellular redox regulation of intracellular reactive oxygen generation, mitochondrial function and lipid turnover in cultured human adipocytes. PloS One. 2016;11(10):e0164011. doi.org/10.1371/journal.pone.0164011

31. Mirończuk-Chodakowska I, Witkowska AM, Zujko ME. Endogenous non-enzymatic antioxidants in the human body. Adv Med Sci. 2018; 63(1):68-78.

32. Mansour RB, Lassoued S, Gargouri B, et al. Increased levels of autoantibodies against catalase and superoxide dismutase associated with oxidative stress in patients with rheumatoid arthritis and systemic lupus erythematosus. Scand J Rheumatol. 2008;37(2):103-108.

33. Shah D, Wanchu A, Bhatnagar A. Interaction between oxidative stress and chemokines: Possible pathogenic role in systemic lupus erythematosus and rheumatoid arthritis. Immunobiology. 2011;216(9):1010-1017.

34. Fukuda W, Omoto A, Oku S, et al. Contribution of rheumatoid arthritis disease activity and disability to rheumatoid cachexia. Mod Rheumatol. 2010;20(5):439-443.

35. Shrivastava AK, Pandey A. Inflammation and rheumatoid arthritis. J Physiol Biochem. 2013;69:335-347.

36. Wei ST, Sun YH, Zong SH, Xiang YB. Serum levels of IL- 6 and TNF-a may correlate with activity and severity of rheumatoid arthritis. Med Sci Monit, 2015;21:4030-4038.

37. Choi HK, Liu S, Curhan G. Intake of purine-rich foods, protein, and dairy products and relationship to serum levels of uric acid: The Third National Health and Nutrition Examination Survey. Arthritis Rheum. 2005;52(1):283-289.

38. Garcimartín A, Merino JJ, Santos-López JA, et al. Silicon as neuroprotector or neurotoxic in the human neuroblastoma SH-SY5Y cell line. Chemosphere. 2015;135: 217-224.

39. Sautin YY, Johnson RJ. Uric acid: The oxidant-antioxidant paradox. Nucleosides Nucleotides Nucleic Acids. 2008;27(6):608-619.

40. Shalaby EA, Shanab SMM. Antioxidant compounds, assays of determination and mode of action. Afr J Pharm Pharmacol. 2013;7:528-539.

41. González-Muñoz MJ, Garcimartan A, Meseguer I, et al. Silicic acid and beer consumption reverses the metal imbalance and the prooxidant status induced by aluminum nitrate in mouse brain. J Alzheimers Dis. 2017;56(3):917-927.

42. Jarosz M. Normy żywienia dla populacji polskiej - nowelizacja (Polish Dietary Reference Intakes - revision). Warsaw: National Food and Nutrition Institute; 2017. (in Polish) https://ncez.pl/upload/normynet-3393.pdf. Accessed August 21, 2018.

43. King SM, Donangelo CM, Knutson MD, et al. Daily supplementation with iron increases lipid peroxidation in young women with low iron stores. Exp Biol Med. 2008;233(6):701-707.

44. Nielsen FH. Silicon deprivation does not significantly modify the acute white blood cell response but does modify tissue mineral distribution response to an endotoxin challenge. Biol Trace Elem Res. 2010:135(1-3):45-55.

45. Gonzalez-Munoz MJ, Meseguer I, Sanchez-Reus MI, et al. Beer consumption reduces cerebral oxidation caused by aluminum toxicity by normalizing gene expression of tumor necrotic factor alpha and several antioxidant enzymes. Food Chem Toxicol. 2008;46(3): 1111-1118. 